\title{
Arterial Stiffness in Aortic Stenosis: Relationship with Severity and Echocardiographic Procedures Response
}

\author{
Giuseppe Bruschi $^{1}$ - Alessandro Maloberti ${ }^{2,3}$ - Paola Sormani ${ }^{2,3}$ - Giulia Colombo ${ }^{2,3}$. \\ Stefano Nava ${ }^{1}$ - Paola Vallerio ${ }^{3}$ - Francesca Casadei ${ }^{3} \cdot$ Jolie Bruno $^{2}$. \\ Antonella Moreo $^{3}$ - Bruno Merlanti ${ }^{4}$ - Claudio Russo ${ }^{4} \cdot$ Fabrizio Oliva $^{1}$. \\ Silvio Klugmann ${ }^{1} \cdot$ Cristina Giannattasio $^{2,3}$
}

Received: 26 October 2016/Accepted: 24 December 2016

(C) Springer International Publishing Switzerland 2017

\begin{abstract}
Introduction Aortic stenosis (AS) is more than only a degenerative disease, it could be also an atheroscleroticlike process involving the valve instead of the vessels. Little is known about the relation of arterial stiffness and AS.

Aim We sought to determine wether pulse wave velocity (PWV), is related to AS severity and to the procedures response, both as surgical aortic-valve-replacement (AVR) and trascatheter-aortic-valve-implantation (TAVI).

Methods 30 patients with severe AS were treated (15 AVR, 15 TAVI). Before the procedures ( $\mathrm{t} 0)$ and after 1 week (t1) echocardiography and PWV were evaluated.

Results On the whole population, subjects with higher PWV showed higher transvalvular pressure gradient at baseline (mean: $56.5 \pm 15.1$ vs $45.4 \pm 9.5$; peak: $93.3 \pm 26.4$ vs $73.3 \pm 14.9, p=0.02)$ and, a significantly greater response to the procedures (mean: $-42.9 \pm 17.2 \mathrm{vs}$ $-27.9 \pm 10.1$, peak: $-68.7 \pm 29.2$ vs $-42.8 \pm 16.4$, $\mathrm{p}=0.02$ ). When the two different procedures groups were separated, data were confirmed only in the TAVI subgroup.
\end{abstract}

Cristina Giannattasio

cristina.giannattasio@unimib.it

1 Cardiology I, “A.De Gasperis” Department, Niguarda Ca Granda Hospital, Milan, Italy

2 Health Science Department, Milano-Bicocca University, Milan, Italy

3 Cardiologia IV, Dipartimento A.De Gasperis, Ospedale Niguarda Ca Granda, Piazza Ospedale Maggiore 3, 20159 Milan, Italy

4 Cardiosurgery, “A.De Gasperis” Department, Niguarda Ca Granda Hospital, Milan, Italy
Conclusions In patients undergoing procedures for AS, PWV is correlated with transvalvular gradient and, in TAVI subjects, is able to predict the echocardiographic response. Baseline evaluation of PWV in patients candidates to TAVI can help the selection of subjects, even if larger and longer studies are needed before definitive conclusion can be drawn.

Keywords Aortic stenosis - Aortic valve replacement . Transcatheter aortic valve implantation - Pulse wave velocity $\cdot$ Arterial stiffness

\section{Introduction}

Aortic stenosis (AS) is a common valve disease that affect $2 \%$ of the population aged more than 65 years reaching the prevalence of $10 \%$ in patients over 80 years old $[1,2]$.

It is frequently asymptomatic but, with the increase of the pressure gradient, and so of the severity of the stenosis, it can determine angina pectoris, syncope, congestive heart failure and sudden cardiac death [1].

In the past AS has been considered as a degenerative disease, but evidences exists that it could be an atherosclerotic-like process involving the valve instead of the vessels. In fact both diseases share some characteristics: they present the same risk factors [3-5] and pathological finding, particularly regarding the similarities between the aortic leaflets and the vascular atherosclerotic plaque $[6,7]$.

The increase in collagen and calcium seen in aortic stenotic valve leads, in the arterial tree, to a vessels stiffening process [8]. Stiffened vessels determine changes in central haemodynamic with an increase in reflected wave velocity that return to the heart in late systole instead of diastole worsening ventricular-vascular coupling and 
inducing an additional load to the left ventricle [9]. This can further deteriorate the heart function in frail patients such as the one with AS, in this situation even a slight increase in afterload is sufficient to determine an excessive myocardial oxygen demand, to impair ventricular relaxation and to determine subendocardial ischemia [10].

Surgical aortic valve replacement (AVR) is considered the gold standard for the management of patients with symptomatic severe AS [1, 2], however, transcatheter aortic valve implantation (TAVI) is increasingly used as an option for patients at very high surgical risk [11, 12].

Since they share the same pathological process it might be expected that arterial stiffness evaluation could predict the severity of AS as well as the response to the procedures. Nevertheless to date little is know about the relation of arterial stiffness and AS [14-19]. With this background in mind we sought to determine wether Pulse Wave Velocity (PWV), i.e. the gold standard for arterial stiffness evaluation, is related to AS severity and to the procedures response, both as AVR and TAVI.

\section{Materials and Methods}

\subsection{Study Population}

Patients with symptomatic severe AS undergoing AVR or TAVI at Niguarda Hospital (Milan, Italy), between June 2012 and April 2013 were recruited. Subjects agreed to participate in the study after being informed of its nature and purpose. The study protocol complies with the Declaration of Helsinki (as revised in 2004) [20] and was approved by the Ethics Committee of the Institution involved.

For both groups exclusion criteria were an age less than 18 years, pregnancy, and atrial fibrillation (AF) at the enrollment visit. Patients were evaluated at time 0 (the day before the procedures) and after 1 week from the AVR or TAVI (time 1). The collected data at time 0 included a full medical history (on $\mathrm{CV}$ risk factors including overweight, smoking habit, diabetes mellitus, history of previous CV events and CV and antihypertensive drugs consumption), blood samples with routine chemistry assessment (serum creatinine, glucose and total and fractionated cholesterol) and a complete physical examination including height and body weight, which allowed calculation of body mass index (BMI$\mathrm{Kg} / \mathrm{m}^{2}$ ). After $10 \mathrm{~min}$ of rest in the supine position, heart rate (HR) and brachial blood pressure (BP) was measured 3 times from the right arm using an oscillometric device (OMRON Healthcare Europe, Hoofddorp, The Netherlands). Finally, keeping the subject in the same position, BP measurements was followed by PWV and trans-thoracic echocardiographic evaluation that were repeated after one week from the procedures.

Glomerular filtration rate was estimated (eGFR) by the modification of diet in renal disease (MDRD) equation [21].

\subsection{Pulse Wave Velocity}

Aortic stiffness was evaluated by PWV between the carotid and the femoral artery of the right side with the patient in the supine position. The pressure pulse waveforms were simultaneously obtained at the two arterial sites using an automatic device (Complior, Colson; Alam Medical, Paris, France) and their distance calculated by taking the distance between hip and neck via a rigid ruler. PWV was automatically provided as the ratio (meters per second) of the delay between the feet of the pressure wave at the second vs the first point and the distance between the two vessels. Distance measurements was corrected by a 0.8 factor accordingly to the PWV measurement methods consensus documents which states to use the subtraction methods instead of the direct one when assessing the distance between the two measurements points [23]. Each data acquisition included at least 10 consecutive cardiac beats for the information to include the complete respiratory cycle. The mean of two acquisitions was used for the analysis.

In our laboratory the intra-session within- and betweenoperator variability of PWV amounts, respectively to a coefficient of variation of the mean value of 2 and to $4 \%$, the corresponding value for the inter-session between-operator variability being $4 \%$.

\subsection{Echocardiography}

Two-dimensional echocardiograms were performed by an experienced cardiologist using a dedicated ultrasound machine (SONOS 5500; Philips Healthcare, Andover, Massachusetts, USA with an ultrasound transducer of 2.5 MHz.). Two-dimensional high frame rate gray-scale loops of four-chamber, two-chamber, and three-chamber views with average frame rate of 90 frames per second were used in order to measure left ventricular end-diastolic diameter (LVEDD), inter-ventricular septum, posterior wall thickness and ejection fraction (EF) by the simpson method. A complete study of the aortic valve including morphology and transvalvular gradients were also done before and after the procedures following standard methods [24]. From the apical five-chamber view, the peak velocity beneath the aortic valve was assessed by pulsed Doppler, and the peak velocity across the valve was assessed using continuous wave Doppler. Pressure gradients were calculated using the modified Bernoulli equation. AV leaflets 
morphology and calcifications were evaluated from the parasternal long- and short-axis views.

\subsection{Procedures}

Surgical intervention for AVR was done during extracorporeal circulation, cardioplegia and full anticoagulation, through median sternotomy and a transverse aortotomy 1-2 cm above the sinotubular junction, the diseased valve was excised and followed by the removal of calcium deposits from the aortic annulus. After that the prosthetic valve (stented) was inserted.

Transcatheter procedures was done in 8 patients with CoreValve and in seven patients with Direct Flow valver implantation. The choice of the valve type was based on operator preference and it was performed using standard protocol [22]. CoreValve is a tricuspid porcine prosthesis (CoreValve; Medtronic Inc, Luxembourg) mounted and sutured within a self-expandable frame made of nitinol while Direct Flow (Direct Flow Medical, Santa Rosa, CA, USA) has conformable sealing rings, which minimizes aortic regurgitation and permits full hemodynamic assessment of valve performance prior to permanent implantation. In both cases a balloon aortic valvuloplasty was performed and subsequently, the prosthetic valve (of a dimension accordingly to aortic annulus) was retrogradely introduced and deployed in the position of the native aortic valve.

\subsection{Statistical Analysis}

Data obtained in each subject were averaged, and individual data were summed and expressed as means $( \pm \mathrm{SD})$, for the whole patients and separately for AVR and TAVI subjects. Between-group differences were assessed by Student $t$, Wilcoxon and $\chi^{2}$ tests (or Fisher exact test when needed) for normally distributed, non-normally distributed and categorical variables, respectively. Pearson's or Spearman's correlation coefficients were used, as appropriate, to test the association between variables. We performed linear regression using the additive model and adjusting for covariates determined by stepwise regression. We used PWV as the dependent variables with age, baseline SBP and peak and mean transvalvular pressure gradient as covariates for multivariate adjustments.

Patients were than divided into subgroup accordingly to the median PWV value $(10.4 \mathrm{~cm} / \mathrm{s}$ for the whole population, $10.2 \mathrm{~cm} / \mathrm{s}$ for the AVR subjects and $10.7 \mathrm{~cm} / \mathrm{s}$ for the TAVI group) and to its increase or decrease at the follow-up visit.

SPSS 13.0 (SPSS, IBM, United States) was used for the statistical analyses and a $\mathrm{p}$ value $<0.05$ was taken as the level of statistical significance.

\section{Results}

\subsection{Baseline Characteristics and Follow-up Evaluation}

Table 1 shows demographic, haemodynamic, biochemical, clinic, PWV and echocardiography data of the enrolled subjects for the whole population and divided in AVR and TAVI subgroups. A total of 30 patients (15 AVR and 15 TAVI) were evaluated with an average age of $79.3 \pm 6.3$ years. They present a consistent prevalence of hypertension $(73.3 \%)$, coronary artery disease (CAD 33.3\%) and diabetes (20.0\%) while only 1 patient actively smokes $(6.6 \%)$. Cardiovascular risk factor were enough controlled as demonstrated by the average BP values (138.0/73.5 $\pm 19.8 /$ $18.6 \mathrm{mmHg})$, glycaemia $(94.0 \pm 18.5 \mathrm{mg} / \mathrm{dL})$ and total and LDL cholesterol $(169.9 \pm 53.8$ and $94.6 \pm 20.1 \mathrm{mg} / \mathrm{dL}$, respectively). Regarding AS, peak and mean transvalvular pressure gradient were $84.4 \pm 23.9$ and $51.1 \pm 13.6$, respectively suggestive of a severe stenosis. Finally PWV showed an average value of $11.0 \pm 3.6 \mathrm{~cm} / \mathrm{s}$.

When baseline characteristics of the two different groups were compared, TAVI patients were older $(82.1 \pm 4.9$ vs $76.5 \pm 6.4$ years, $p=0.01)$ and present more frequently dislipidemia (60.0 vs $33.3 \%)$ and diabetes (33.3 vs 6.6\%). All the remaining variables including BP values, transvalvular pressure gradient, $\mathrm{EF}$ and $\mathrm{PWV}$ were superimposable between the two groups.

One week after procedures (t1) clinic variables, PWV and echocardiography were repeated (Table 1). In the whole population a decrease in SBP with a slightly increase in HR was shown at time 1 associated with a good echocardiographic response to the procedures with a statistically significant average decrease in transvalvular pressure gradient of $-59.0 \pm 27.8$ and $-36.9 \pm 16.3$ for the peak and mean gradient, respectively $(\mathrm{p}<0.01$ for peak and mean transvalvular gradient at follow-up vs baseline evaluation). On the contrary EF and PWV did not show any change at the follow-up evaluation.

When patients were divided accordingly to the two different procedures AVR subjects shown a significant decrease in SBP associated with an increase in HR that was not present in TAVI group. Instead both groups showed a superimposable decrease in aortic transvalvular pressure gradient as well as no significant changes in EF and PWV values.

\subsection{Pulse Wave Velocity Analysis}

Table 2 presents the data of the enrolled subjects divided into high and low PWV accordingly to its median value. In the whole population high vs low PWV subjects were more 
Table 1 Demographic, haemodynamic, biochemical, clinic, PWV and echocardiography data of the enrolled subjects for the whole population and for AVR and TAVI groups

\begin{tabular}{|c|c|c|c|c|}
\hline & All patients & AVR & TAVI & $\mathrm{p}$ value (AVR vs TAVI) \\
\hline Number & 30 & 15 & 15 & \\
\hline Age (years, SD) & $79.3(6.3)$ & $76.5(6.4)$ & $82.1(4.9)$ & 0.01 \\
\hline Female $(\%)$ & 50.0 & 40.0 & 60.0 & 0.12 \\
\hline SBP t0 (mmHg) & $138.0(19.8)$ & $141.7(20.6)$ & $134.3(18.9)$ & 0.31 \\
\hline SBP t1 (mmHg) & $129.2(18.6)^{*}$ & $124.0(19.3)^{*}$ & $134.5(17.1)$ & 0.62 \\
\hline DBP t0 (mmHg) & $73.5(8.7)$ & $75.0(9.8)$ & $72.0(7.5)$ & 0.35 \\
\hline DBP t1 (mmHg) & $70.3(10.4)$ & $68.2(11.8)$ & $72.3(8.58)$ & 0.28 \\
\hline HR t0 (bpm) & $66.8(14.6)$ & $65.5(10.7)$ & $68.1(18.1)$ & 0.62 \\
\hline HR t1 (bpm) & $73.1(9.3)^{*}$ & $77.7(9.2)^{*}$ & $68.5(7.3)$ & 0.07 \\
\hline BMI $\left(\mathrm{kg} / \mathrm{m}^{2}\right)$ & $25.2(3.6)$ & $25.1(3.8)$ & $25.2(3.5)$ & 0.96 \\
\hline Hypertension (\%) & 73.3 & 73.3 & 73.3 & 1 \\
\hline Dislipidemia (\%) & 46.6 & 33.3 & 60.0 & 0.04 \\
\hline Diabetes mellitus (\%) & 20.0 & 6.6 & 33.3 & 0.002 \\
\hline CAD $(\%)$ & 33.3 & 26.6 & 40.0 & 0.06 \\
\hline Actual smoking (\%) & 3.3 & 0 & 6.6 & - \\
\hline Glycemia (mg/dL) & $94.0(18.5)$ & $92.1(8.2)$ & $69.0(25.7)$ & 0.59 \\
\hline Total cholesterol (mg/dL) & $169.9(53.8)$ & $184.8(18.6)$ & $154.9(72.6)$ & 0.22 \\
\hline LDL cholesterol (mg/dL) & $94.6(20.1)$ & $105.3(16.7)$ & $83.8(52.1)$ & 0.23 \\
\hline GFR (mL/min) & $65.0(24.8)$ & $70.6(16.4)$ & $59.5(30.6)$ & 0.21 \\
\hline EF t0 & $0.56(0.15)$ & $0.52(0.18)$ & $0.59(0.11)$ & 0.32 \\
\hline$\Delta \mathrm{EF} \mathrm{t} 1$ & $0.0(0.2)$ & $0.0(0.2)$ & $0.0(0.07)$ & 0.21 \\
\hline Peak transvalvular grad. (mmHg) & $84.4(23.9)$ & $81.8(17.0)$ & $86.5(28.7)$ & 0.62 \\
\hline Mean transvelvular grad. med ( $\mathrm{mmHg}$ ) & $51.1(13.6)$ & $50.1(9.4)$ & $52.1(16.9)$ & 0.69 \\
\hline$\Delta$ Peak transvalvular grad. $(\mathrm{mmHg})$ & $-59.0(27.8)^{\#}$ & $-55.4(18.1)^{\#}$ & $-61.5(33.5)^{\#}$ & 0.61 \\
\hline$\Delta$ Mean transvelvular grad. $(\mathrm{mmHg})$ & $-36.9(16.3)^{\#}$ & $-35.5(7.9)^{\#}$ & $-38.0(21.1)^{\#}$ & 0.71 \\
\hline PWV t0 (cm/s) & $11.0(3.6)$ & $11.2(4.3)$ & $10.9(2.8)$ & 0.79 \\
\hline$\Delta \mathrm{PWV} \mathrm{t} 1(\mathrm{~cm} / \mathrm{s})$ & $0.4(2.4)$ & $0.4(2.4)$ & $0.3(2.5)$ & 0.97 \\
\hline
\end{tabular}

$S B P$ systolic blood pressure, $D B P$ diastolic blood pressure, $H R$ heart rate, $B M I$ body mass index, $C A D$ coronary artery disease, $L D L$ low density lypoprotein, $G F R$ glomerular filtration rate, $E F$ ejection fraction, $P W V$ pulse wave velocity

$* \mathrm{p}<0.05$ for follow-up vs baseline evaluation

\# $\mathrm{p}<0.01$ for peak and mean transvalvular gradient at follow-up vs baseline evaluation

frequently female and diabetic and present higher baseline SBP, their mean PWV values were $13.4 \pm 3.6$ and $8.7 \pm 1.3 \mathrm{~cm} / \mathrm{s}$, respectively. Of note they present higher transvalvular mean and peak pressure gradient at baseline (mean: $56.5 \pm 15.1$ vs $45.4 \pm 9.5$, peak: $93.3 \pm 26.4$ vs $73.3 \pm 14.9, \mathrm{p}=0.02$ for both comparison; for high vs low PVW group, respectively-Fig. 1) and, moreover, a significantly better echocardiographic response to the procedures (mean: $-42.9 \pm 17.2$ vs $-27.9 \pm 10.1$; peak: $-68.7 \pm 29.2$ vs $-42.8 \pm 16.4, \mathrm{p}=0.02$ for both comparison-Fig. 2).

When patients were divided accordingly to the two different procedures in AVR subgroup high PWV subjects did not differ for any variables except for PWV baseline values when compared to low PWV patients (Figs. 1,2).
In TAVI subgroup high PWV subjects were more frequently female and diabetic and present higher baseline SBP. Of note high PWV subjects respond significantly better to the procedure when compared to low PWV patients (mean: $-48.6 \pm 22.3$ vs $-23.8 \pm 5.3, \mathrm{p}=0.02$; peak: $-76.6 \pm 37.7 \mathrm{vs}-41.3 \pm 9.2, \mathrm{p}=0.04$; for high vs low PVW, respectively_Fig. 2).

Finally we divided patients in two subgroup depending on whether they increase or decrease they PWV values at follow-up evaluation (Table 3). In all subjects and AVR and TAVI subgroups no differences where seen between the two subgroups particularly regarding the procedures response.

As shown in Table 4, at univariate correlation analysis in all subjects baseline PWV correlates with t0 SBP 


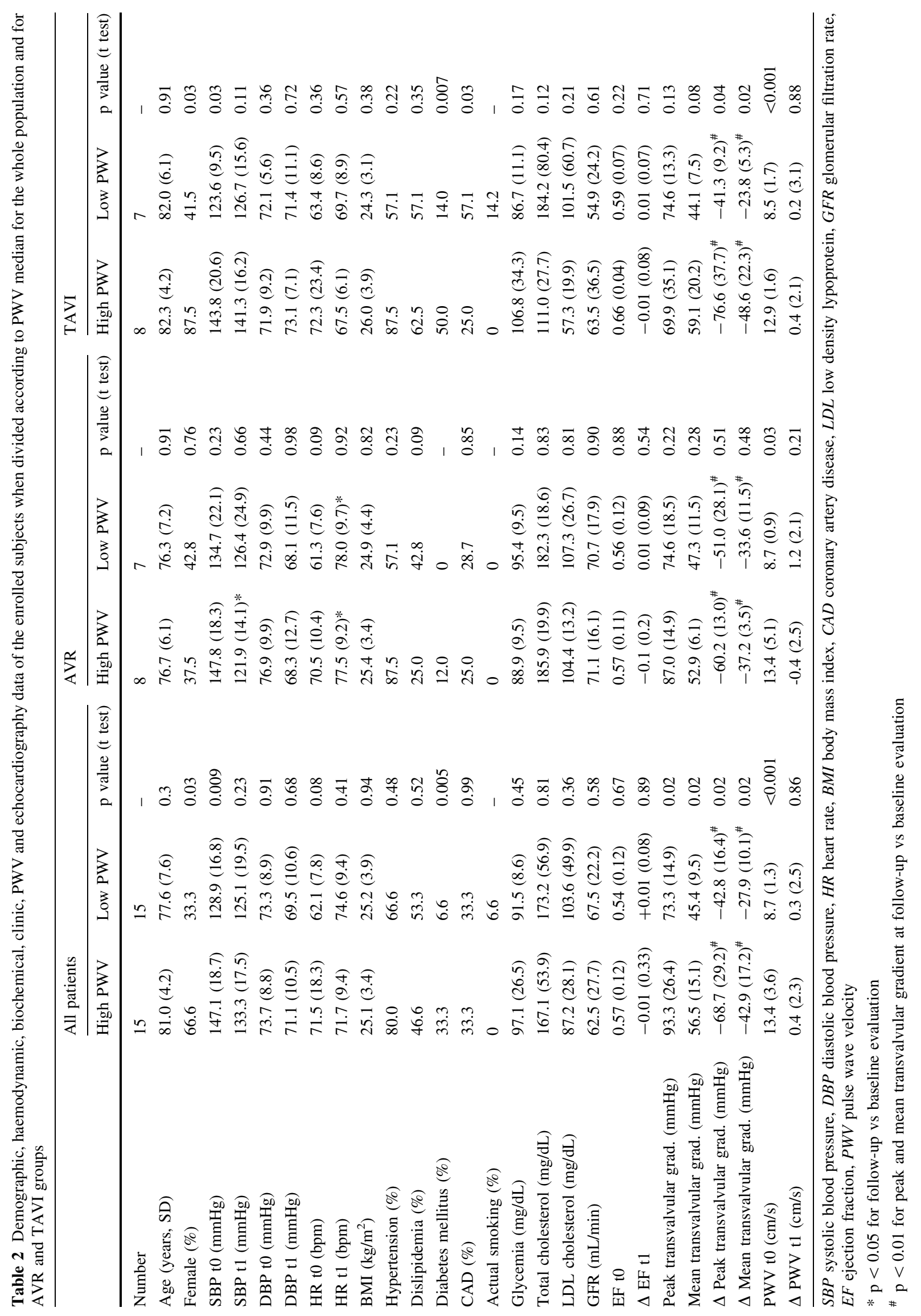




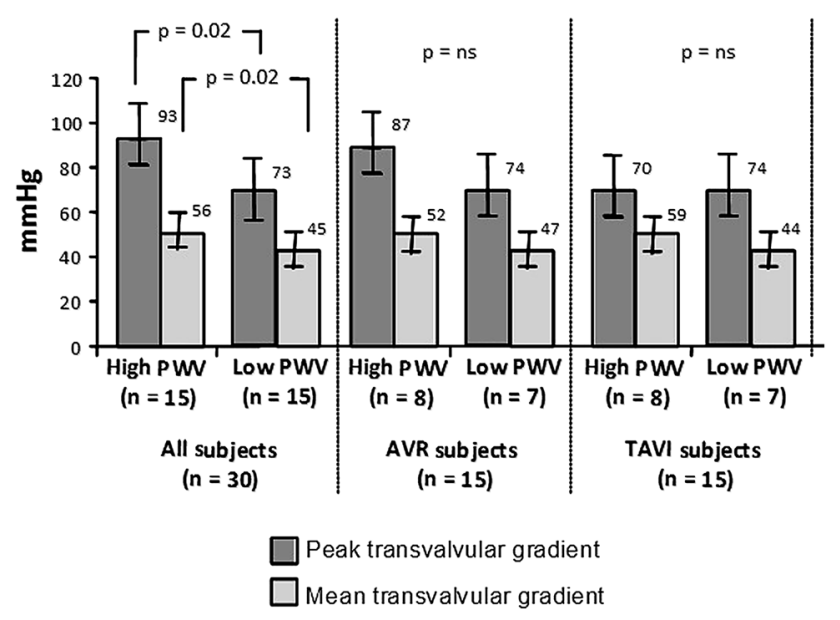

Fig. 1 Peak and mean transvalvular gradient when subjects are divided according to high and low PWV values for the whole population and for AVR and TAVI groups

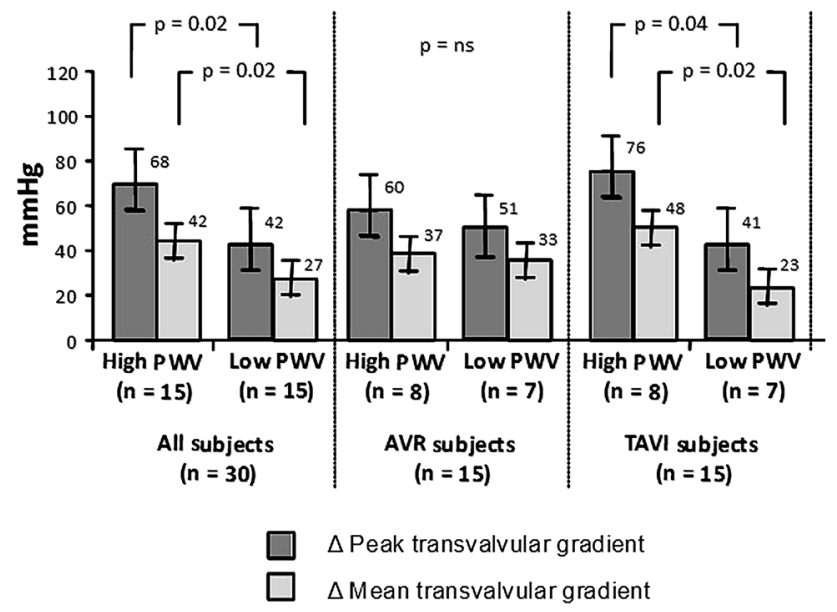

Fig. $2 \Delta$ Peak and mean transvalvular gradient when subjects are divided according to high and low PWV values for the whole population and for AVR and TAVI groups

$(\mathrm{r}=0.39, \mathrm{p}=0.03), \mathrm{t} 0$ peak and mean transvalvular gradient $(\mathrm{r}=0.40, \mathrm{p}=0.03$ and $\mathrm{r}=0.37, \mathrm{p}=0.04$, respectively) and also with $\Delta$ peak and mean transvalvular gradient $(\mathrm{r}=-0.44, \mathrm{p}=0.02$ and $\mathrm{r}=-0.39, \mathrm{p}=0.04$ respectively). In this quite old group of patients, no correlation was seen with age, DBP, cholesterol, glycemia and EF. When patients were divided accordingly to the two different procedures no correlation was seen in AVR patients while all the correlation were confirmed, and further strengthened in TAVI subjects ( $\mathrm{t} 0$ peak transvalvular gradient: $\mathrm{r}=0.55, \mathrm{p}=0.03$; t0 mean transvalvular gradient and $\mathrm{r}=0.58, \mathrm{p}=0.02 ; \Delta$ peak transvalvular gradient: $\mathrm{r}=-0.70, \mathrm{p}=0.004 ; \Delta$ mean transvalvular gradient: $\mathrm{r}=-0.72 ; \mathrm{p}=0.003) . \Delta \mathrm{PWV}$ did not show any significant correlations.

The relationship between $\Delta$ peak and mean transvalvular gradient and PWV resist at the multivariate analysis in
TAVI subjects with a $r^{2}$ of 0.66 and 0.62 , respectively $(\mathrm{p}<0.05)$.

\section{Discussion}

Our study provides evidence of a correlation between baseline PWV and peak and mean transvalvular pressure gradient (representative of AS severity) in patients with symptomatic high grade AS. Furthermore we demonstrate that PWV is able to predict the procedure response in TAVI subgroups subjects.

Only three previous work evaluated arterial stiffness relation with AS severity. Liu et al. [17] found a significant association between increased aortic pressure gradient and high PWV values. By contrast, Celik et al. [18] and Kidher et al. [19] did not found this correlation.

By demonstrating this relation our study supports the hypothesis that AS and arterial stiffness are different spectrum of the same atherosclerotic disease. Since there were no important patients enrollment and technical differences between our and the other cited studies only a larger study can probably drawn definitive conclusion regarding correlation between arterial stiffness and AS.

We reassess arterial stiffness one week after procedures and we found that average PWV did not change in the early post-operative period both in surgical AVR and TAVI subjects. Previous works on the field used a different technique, i.e., the $\beta$ stiffness index of the ascending aorta with echocardiographic evaluation. Similarly to arterial distensibility it is a vascular stiffness index that quantify changes in arterial diameter in response to blood pressure changes from diastole to systole [25]. Accordingly to expert consensus [8], different stiffness index provide different pathophysiological and therapeutical information, so, in this case, results are not of simple interpretation. Evidence exist of an impairment of the $\beta$ stiffness index of the ascending aorta in the first post-operative period (1 week) in surgical AVR subjects [16] that is not confirmed in TAVI patients [15]. In both procedures long-term follow-up shown a normalization or an improvement in distensibility of the ascending aorta $[13,16]$. As speculate from the cited works $[15,16]$, the impairment in the early post-operative period is probably determined by an "aortic stunning" during the surgical AVR that is not present in the TAVI subjects.

Dissimilarities from the cited works could be explained by the different methodological assessment. In fact PWV (femoral to carotid) evaluate all the aortic vessel, instead previous studies assess only ascending aorta $\beta$ index. Ascending aorta is the vessel that is directly influenced by the two procedures and so its evaluation permit to shown earlier changes. 


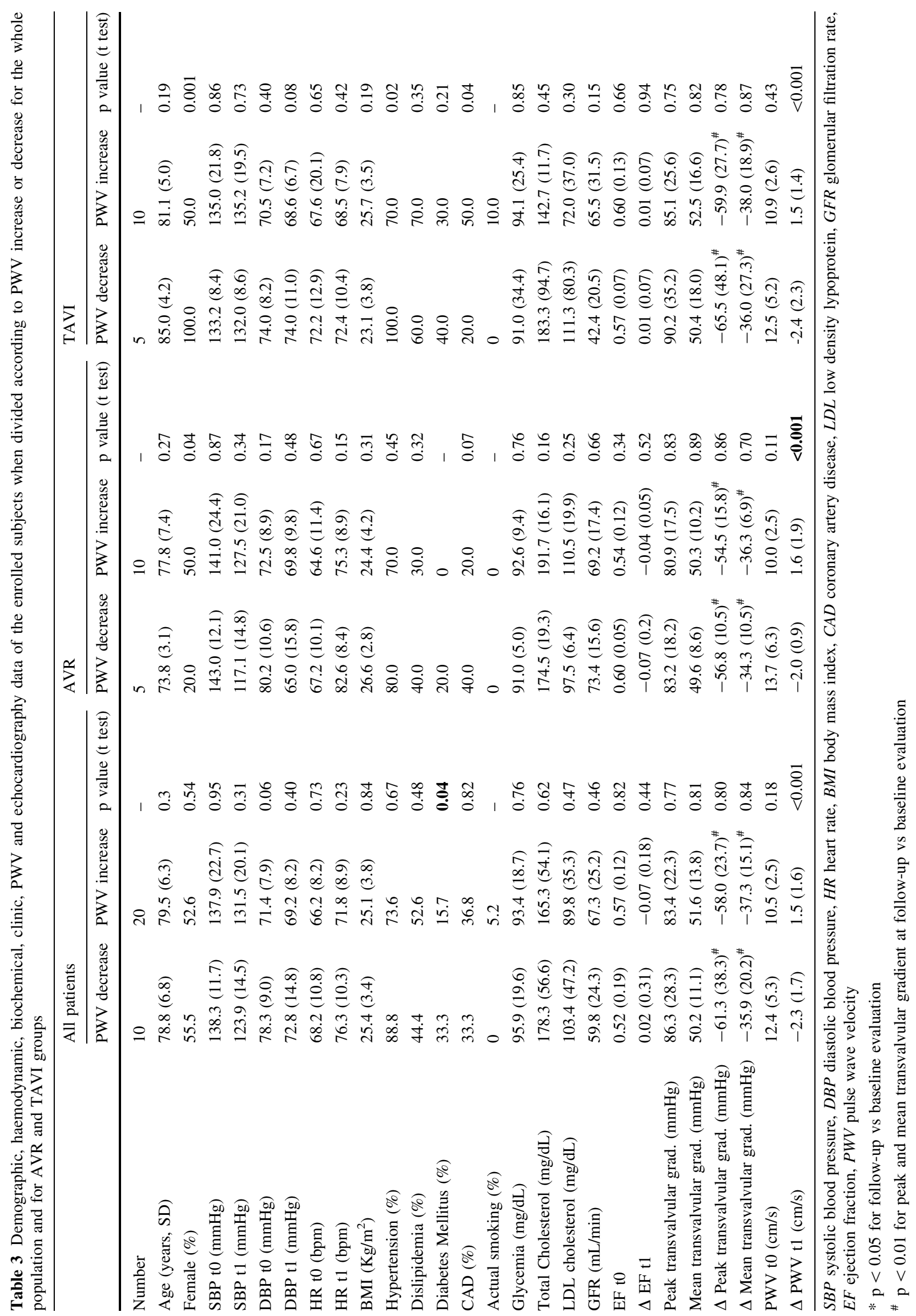


Table 4 PWV correlations with transvalvular gradient in whole population and for AVR and TAVI groups

\begin{tabular}{|c|c|c|c|c|c|c|}
\hline & \multicolumn{2}{|c|}{ All patients } & \multicolumn{2}{|l|}{ AVR } & \multicolumn{2}{|l|}{ TAVI } \\
\hline & $\mathrm{r}$ & $\mathrm{p}$ & $\mathrm{r}$ & $\mathrm{p}$ & $\mathrm{r}$ & $\mathrm{p}$ \\
\hline Peak transvalvular grad. (mmHg) & 0.40 & $\mathbf{0 . 0 3}$ & 0.54 & 0.06 & 0.55 & $\mathbf{0 . 0 3}$ \\
\hline Mean transvalvular grad. (mmHg) & 0.37 & 0.04 & 0.47 & 0.09 & 0.58 & 0.02 \\
\hline$\Delta$ Peak transvalvular grad. $(\mathrm{mmHg})$ & -0.44 & 0.02 & -0.33 & 0.34 & -0.7 & 0.004 \\
\hline$\Delta$ Mean transvalvular grad. $(\mathrm{mmHg})$ & -0.39 & 0.04 & -0.46 & 0.15 & -0.72 & 0.003 \\
\hline
\end{tabular}

In our opinion, the most important finding of our work, however, is the possibility of the PWV to predict the echocardiographic response to the aortic valve replacement procedures. Both surgical AVR and TAVI subgroup shown a good response to the procedures but the finding of a better response in patients with higher PWV was confirmed only in TAVI subjects. Although average PWV values did not change, in some patient it increases and in some others it decreases. When we divided patients accordingly to their increased/decreased PWV values no differences between groups were seen demonstrating that baseline values are more important than their variance in the early post-operative period. Although patients with higher PWV showed better TAVI procedures response they are probably at higher risk of CV complication despite we did not analyze this point due to the few number of events.

Only one previous study evaluated PWV in AVR subjects showing its correlation with NYHA class both pre and post-operatively but not with proBNP and EF [19]. Other analysis from the same study found that higher PWV was correlated with poor quality of life [26] and cognitive dysfunction [27] both pre and post-operatively and that it does not correlate with post-operatively acute kidney failure, a well know surgical AVR complication [28].

This is the first time that carotid-femoral PWV is evaluated in AS patients undergoing both AVR and TAVI. These procedures presents different indication and their influences on aortic root is very different. Our finding of its correlation with echocardiographic response only in TAVI subjects probably indicates that in surgical AVR patients other factors determine the response. These are likely correlated with the surgical procedure such as the cardiosurgical and anesthesiological variables, the need of ematic transfusion or the drop in BP and HR post-operatively.

Unfortunately we did not assess systematically NYHA class on long-term follow-up so we can only speculate on echocardiographic short-term procedures response instead of the clinical one.

Our study presents some limitations. First of all, similarly to previous studies on AS, the present one was limited by the small number of patients and so interpretation of the results of the current study require caution. Furthermore the limited number of subjects made some subgroups small, limiting the power to detect associations or changes.
Thirdly, changes in drug treatment were not registered and so we were unable to take into account effect of changes when data were analyzed. The principal limitation of our study, as we have already highlighted, is the absence of clinical response data (i.e. NYHA class) and laboratory data (i.e. proBNP assessment) as well as the short-term follow-up data that doesn't allow to draw conclusion on a longer time, even if long follow-up could be difficult since the population is already very old at the baseline evaluation.

\section{Conclusions}

Higher PWV is correlated with higher transvalvular pressure gradient in AS subjects, that it doesn't change in the early post-operative period and, finally, that it is able to predict the echocardiographic procedure response in TAVI subgroup. Baseline evaluation of arterial stiffness in patients candidates to TAVI can help correctly selects subjects, even if larger and longer follow-up studies are needed before definitive conclusion can be drawn.

\section{Compliance with ethical standards}

Conflict of interest All the authors declare that they have no conflict of interest.

Ethical approval All procedures performed in studies involving human participants were in accordance with the ethical standards of the institutional and/or national research committee and with the 1964 Helsinki declaration and its later amendments or comparable ethical standards.

Funding This research did not receive any specific grant from funding agencies in the public, commercial, or not-for-profit sectors.

Informed consent Informed consent was obtained from all individual participants included in the study.

\section{References}

1. Rashedi N, Otto CM. Aortic Stenosis: changing diseae concepts. J Cardiovasc Ultrasound. 2015;23(2):59-69.

2. Czarny MJ, Resar JR. Diagnosis and management of valvular aortic stenosis. Clin Med Insights Cardiol. 2014;8:15-24. 
3. Poggianti E, Venneri L, Chubuchny V, Jambrik Z, Baroncini LA, Picano E. Aortic valve sclerosis is associated with systemic endothelial dysfunction. J Am Coll Cardiol. 2003;41:136-41.

4. Agmon Y, Khandheria BK, Jamil Tajik A, Seward JB, Sicks JD, Fought AJ, O'Fallon WM, Smith TF, Wiebers DO, Meissner I. Inflammation, infection, and aortic valve sclerosis; Insights from the Olmsted County (Minnesota) population. Atherosclerosis. 2004;174(2):337-42.

5. Chandra HR, Goldestein JA, Choudhary N, O'Neill CS, George PB, Gangasani SR, Cronin L, Marcovitz PA, Hauser AM, O'Neill WW. Adverse outcome in aortic sclerosis is associated with coronary artery disease and inflammation. J Am Coll Cardiol. 2004;43:169-75.

6. Otto CM, Kuusisto J, Reichenbach DD, Gown AM, O'Brien KD. Characterization of the early lesion in "degenerative" valvular aortic stenosis: histological and immunohistochemical studies. Circulation. 1994;90:844-53.

7. Lee YS, Chou YY. Pathogenetic mechanism of senile calcific aortic stenosis: the role of apoptosis. Chin Med J. 1998;111:934-9.

8. Laurent S, Cockcroft J, Van Bortel L, Boutouyrie P, Giannattasio C, Hayoz D, Pannier B, Vlachopoulos C, Wilkinson I, StruijkerBoudier H. Expert consensus document on arterial stiffness: methodological issues and clinical applications. Eur Heart J. 2006;27:2588-605.

9. Asanoi H, Sasayama S, Kameyama T. Ventriculoarterial coupling in normal and failing heart in humans. Circ Res. 1989;65:483-93.

10. O'Rourke MF. How stiffening of the aorta and elastic arteries leads to compromised coronary flow. Heart. 2008;94:690-1.

11. Vahanian A, Alfieri O, Al-Attar N, Antunes M, Bax J, Cormier B, Cribier A, De Jaegere P, Fournial G, Kappetein AP, Kovac J, Ludgate S, Maisano F, Moat N, Mohr F, Nataf P, Piérard L, Pomar JL, Schofer J, Tornos P, Tuzcu M, van Hout B, Von Segesser LK, Walther T. Transcatheter valve implantation for patients with aortic stenosis: a position statement from the European Association of Cardio-Thoracic Surgery (EACTS) and the European Society of Cardiology (ESC), in collaboration with the European Association of Percutaneous Cardiovascular Interventions (EAPCI). Eur Heart J. 2008;29(11):1463-70.

12. Sarkar K, Sarkar M, Ussia GP. Current status of transcatheter aortic valve replacement. Med Clin North Am. 2015;99(4):805-33.

13. Nemes A, Galema TW, Geleijnse ML, Soliman OI, Yap SC, Anwar AM, ten Cate FJ. Aortic valve replacement for aortic stenosis is associated with improved aortic distensibility at longterm follow-up. Am Heart J. 2007;153(1):147-51.

14. Nemes A, Galema TW, Soliman OI, Bogers AJ, ten Cate FJ, Geleijnse ML. Improved aortic distensibility after aortic homograft root replacement at long-term follow-up. Int $\mathrm{J}$ Cardiol. 2009;136(2):216-9.

15. Vavuranakis M, Vrachatis D, Boudoulas H, Papaioannou TG, Moldovan C, Kariori MG, Kalogeras KI, Pietri PG, Tentolouris C, Stefanadis C. Effect of transcatheter aortic valve implantation on the ascending aorta's elasticity. Clin Res Cardiol. 2012;101:895-9.

16. Barbetseas J, Alexopoulos N, Brili S, Aggeli C, Marinakis N, Vlachopoulos C, Vyssoulis G, Stefanadis C. Changes in aortic root function after valve replacement in patients with aortic stenosis. Int J Cardiol. 2006;110(1):74-9.

17. Liu PY, Tsai WC, Lin CC, Hsu CH, Haung YY, Chen JH. Invasive measurements of pulse wave velocity correlate with the degree of aortic valve calcification and severity associated with matrix metalloproteinases in elderly patients with aortic valve stenosis. Clin Sci (Lond). 2004;107(4):415-22.

18. Celik S, Durmuş I, Korkmaz L, Gedikli O, Kaplan S, Orem C, Baykan M. Aortic pulse wave velocity in subjects with aortic valve sclerosis. Echocardiography. 2008;25(10):1112-6.

19. Kidher E, Harling L, Ashrafian H, Naase H, Francis DP, Evans P, Athanasiou T. Aortic stiffness as a marker of cardiac function and myocardial strain in patients undergoing aortic valve replacement. J Cardiothorac Surg. 2014;17(9):102.

20. Word Medical Association Declaration of Helsinki, Ethical principles for Medical Research Involving Human Subjects. http://www.wma.net/e/policy/b3.htm. Accessed on 20 March 2016.

21. Modification of Diet in Renal Disease. Expressing the modification of diet in renal disease study equation for estimating glomerular filtration rate with standardized serum creatinine values. Clin Chem. 2007;53(4):766-72.

22. Vavuranakis M, Voudris V, Vrachatis DA, Thomopoulou S, Toutouzas K, Karavolias G, Tolios I, Sbarouni E, Lazaros G, Chrysohoou C, Khoury M, Brili S, Balanika M, Moldovan C, Stefanadis C. Transcatheter aortic valve implantation, patient selection process and procedure: two centres' experience of the intervention without general anaesthesia. Hellenic J Cardiol. 2010;51(6):492-500.

23. The Reference Values for Arterial Stiffness' Collaboration. Determinants of pulse wave velocity in healthy people and in the presence of cardiovascular risk factors: 'establishing normal and reference values'. Eur Heart J. 2010;31:2338-50.

24. Otto CM, Pearlman AS. Valvular stenosis: diagnosis, quantitation, and clinical approach. In: Textbook of Clinical Echocardiography (1995), pp. 209-242.

25. Maloberti A, Meani P, Varrenti M, Giupponi L, Stucchi M, Vallerio P, Giannattasio C. Structural and functional abnormalities of carotid artery and their relation with EVA phenomenon. High Blood Press Cardiovasc Prev. 2015;22(4):373-9.

26. Kidher E, Harling L, Nihoyannopoulos P, Shenker N, Ashrafian H, Francis DP, Mayet J, Athanasiou T. High aortic pulse wave velocity is associated with poor quality of life in surgical aortic valve stenosis patients. Interact Cardio Vasc Thorac Surg. 2014;19:189-97.

27. Kidher E, Harling L, Sugden C, Ashrafian H, Casula R, Evans P, Nihoyannopoulos P, Athanasiou T. Aortic stiffness is an indicator of cognitive dysfunction before and after aortic valve replacement for aortic stenosis. Interact CardioVasc Thorac Surg. 2014;19:595-604.

28. Kidher E, Harling L, Ashrafian H, Naase H, Chukwuemeka A, Anderson J, Francis DP, Athanasiou T. Pulse wave velocity and neutrophil gelatinase-associated lipocalin as predictors of acute kidney injury following aortic valve replacement. J Cardiothorac Surgery. 2014;9:89. 